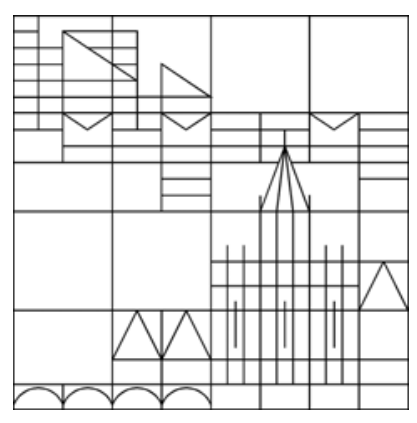

\title{
Numerical Analysis of POD A-Posteriori Error Estimation for Optimal Control
}

\author{
Alina Studinger \\ Stefan Volkwein
}

Konstanzer Schriften in Mathematik

Nr. 296, Februar 2012

ISSN 1430-3558

Konstanzer Online-Publikations-System (KOPS)

URL: http://nbn-resolving.de/urn:nbn:de:bsz:352-184648

(C) Fachbereich Mathematik und Statistik

Universität Konstanz

Fach D 197, 78457 Konstanz, Germany 



\title{
Numerical Analysis of POD A-Posteriori Error Estimation for Optimal Control
}

\author{
Alina Studinger and Stefan Volkwein
}

\begin{abstract}
In this paper a linear-quadratic optimal control problem governed by a parabolic equation is considered. To solve this problem numerically a reduced-order approach based on proper orthogonal decomposition (POD) is applied. The error between the POD suboptimal control and the optimal control of the original problem is controlled by an a-posteriori error analysis. In this paper the authors focus on testing the a-posteriori estimate's validity by means of numerical examples. An intensive study of the consequences of certain choices that can be made within the POD basis determination process is carried out and the findings are discussed.
\end{abstract}

Mathematics Subject Classification (2010). Primary 35K90, 49K20; Secondary $65 \mathrm{~K} 05$.

Keywords. Optimal control, model reduction, proper orthogonal decomposition, a-posteriori error estimates, primal-dual active set strategy.

\section{Introduction}

Optimal control problems for partial differential equation are often hard to tackle numerically because their discretization leads to large scale optimization problems. Therefore, different techniques of model reduction were developed to approximate these problems by smaller ones that are tractable with less effort. Among them, proper orthogonal decomposition (POD) [20] and balanced truncation [4] seem to be most widely used in the context of optimal control. Recently, optimal control problems are also treated by the reduced basis method; we refer, e.g., to $[5,6,17]$.

POD is based on projecting the dynamical system onto subspaces of basis elements that express characteristics of the expected solution. This is

The second author gratefully acknowledges support by the German Science Fund DFG grant VO 1658/2-1 A-posteriori-POD Error Estimators for Nonlinear Optimal Control Problems governed by Partial Differential Equations. 
in contrast to, e.g., finite element techniques, where the elements are not correlated to the physical properties of the system they approximate.

In our present work, POD is applied to linear-quadratic optimal control problems. Linear-quadratic problems are interesting in several respects; in particular, since they occur in each level of sequential quadratic programming (SQP) methods; see, e.g., [19] from a general viewpoint and [12, 20] in the context of multilevel reduced-order approximations. We continue the research on POD a-posteriori error analysis; see [12, 13, 20, 22, 24]. Based on a perturbation argument it is derived how far the suboptimal control, computed on the basis of the POD model, is from the (unknown) exact one. Increasing the number of POD ansatz functions leads to more accurate POD suboptimal controls. This idea turns out to be numerically very efficient. It is also successfully applied for other reduced-order approximations; see [21, 25].

Here, we focus on testing the a-posteriori estimate's validity by means of numerical examples. We intensively study the consequences of certain choices that can be made within the POD basis determination process. Let us summarize the key findings here:

- The estimation is very satisfactory and valuable in terms of accuracy, reliability and efficiency.

- Both the primal-dual active set strategy for solving the control constrained optimal control problem and the a-posteriori error estimation for tracking the error work very well for control box constraints. In case of active constraints, we discover numerical convergence of the active sets which is perfect in case of an "optimal" POD basis (computed from the optimal FE solution) and satisfactory for arbitrary bases.

- In order to obtain good POD suboptimal controls it is not sufficient to solely increase the number of used POD basis functions. Increasing the basis rank needs to be combined with basis update strategies, as for example discussed in $[1,2,15,24]$.

- Enriching the snapshot ensemble by snapshots from the adjoint state is essential to obtain good approximations for the control.

This paper is organized as follows: In Section 2 we introduce the abstract linear-quadratic optimal control problem and review first-order necessary optimality conditions. The POD method, its application to the optimal control problem and the a-posteriori error estimate are explained in Section 3. In Section 4 numerical examples are presented and discussed.

\section{The optimal control problem}

In this section, we introduce a class of linear-quadratic parabolic optimal control problems and recall the associated first-order optimality conditions.

\subsection{Problem formulation}

Let $V$ and $H$ be real, separable Hilbert spaces and suppose that $V$ is dense in $H$ with compact embedding. By $\langle\cdot, \cdot\rangle_{H}$ we denote the inner product in $H$. 
The inner product in $V$ is given by a symmetric bounded, coercive, bilinear form $a: V \times V \rightarrow \mathbb{R}:$

$$
\langle\varphi, \psi\rangle_{V}=a(\varphi, \psi) \quad \text { for all } \varphi, \psi \in V
$$

with associated norm $\|\cdot\|_{V}=\sqrt{a(\cdot, \cdot)}$. By identifying $H$ with its dual $H^{\prime}$ it follows that $V \hookrightarrow H=H^{\prime} \hookrightarrow V^{\prime}$, each embedding being continuous and dense. Recall that for $T>0$ the space $W(0, T)$

$$
W(0, T)=\left\{\varphi \in L^{2}(0, T ; V): \varphi_{t} \in L^{2}\left(0, T ; V^{\prime}\right)\right\}
$$

is a Hilbert space endowed with the common inner product [3]. When the time $t$ is fixed, the expression $\varphi(t)$ stands for the function $\varphi(t, \cdot)$ considered as a function in $\Omega$ only. Let $\mathcal{D}$ be an open and bounded subset in $\mathbb{R}^{m}$ with $m \in \mathbb{N}$. By $U_{\text {ad }}$ we denote the closed, convex and bounded subset

$$
U_{\text {ad }}=\left\{u \in L^{2}(\mathcal{D}) \mid u_{a}(s) \leq u(s) \leq u_{b}(s) \text { for almost all (f.a.a.) } s \in \mathcal{D}\right\},
$$

where $u_{a}, u_{b} \in L^{2}(\mathcal{D})$ satisfy $u_{a} \leq u_{b}$ almost everywhere (a.e.) in $\mathcal{D}$. For $y_{0} \in V, f \in L^{2}(0, T ; H)$ and $u \in U_{\text {ad }}$ we consider the linear evolution problem

$$
\begin{aligned}
\frac{\mathrm{d}}{\mathrm{d} t}\langle y(t), \varphi\rangle_{H}+a(y(t), \varphi) & =\langle(f+\mathcal{B} u)(t), \varphi\rangle_{H} & \text { f.a.a. } t \in[0, T], \forall \varphi \in V, \\
\langle y(0), \varphi\rangle_{H} & =\left\langle y_{0}, \varphi\right\rangle_{H} & \forall \varphi \in V,
\end{aligned}
$$

where $\mathcal{B}: L^{2}(\mathcal{D}) \rightarrow L^{2}(0, T ; H)$ is a continuous, linear operator.

Example 2.1. Let us present an example for (2.2) which will be studied in our numerical experiments. Suppose that $\Omega \subset \mathbb{R}^{2}$, is an open and bounded domain with Lipschitz-continuous boundary $\Gamma=\partial \Omega$. For $T>0$ we set $Q=(0, T) \times \Omega$ and $\Sigma=(0, T) \times \Gamma$. Let $H=L^{2}(\Omega), V=H^{1}(\Omega)$ and $\mathcal{D}=\Sigma$. Then, for given control $u \in L^{2}(\Sigma)$ and initial condition $y_{0} \in V$ we consider

$$
\begin{aligned}
c_{p} y_{t}(t, \boldsymbol{x})-\Delta y(t, \boldsymbol{x}) & =\tilde{f}(t, \boldsymbol{x}) & & \text { f.a.a. }(t, \boldsymbol{x}) \in Q, \\
\frac{\partial y}{\partial n}(t, \boldsymbol{s})+q y(t, \boldsymbol{s}) & =u(t, \boldsymbol{s}) & & \text { f.a.a. }(t, \boldsymbol{s}) \in \Sigma, \\
y(0, \boldsymbol{x}) & =y_{0}(\boldsymbol{x}) & & \text { f.a.a. } \boldsymbol{x} \in \Omega .
\end{aligned}
$$

In (2.3a) we suppose $c_{p}>0, q \geq 0$ and $\tilde{f} \in L^{2}(0, T ; H)$. Setting $f=\tilde{f} / c_{p}$, introducing the bounded bilinear form $a: V \times V \rightarrow \mathbb{R}$ by

$$
a(\varphi, \psi)=\frac{1}{c_{p}} \int_{\Omega} \nabla \varphi(\boldsymbol{x}) \cdot \nabla \psi(\boldsymbol{x}) \mathrm{d} \boldsymbol{x}+\frac{q}{c_{p}} \int_{\Gamma} \varphi(\boldsymbol{s}) \psi(\boldsymbol{s}) \mathrm{d} \boldsymbol{s} \quad \text { for } \varphi, \psi \in V
$$

and the linear, bounded operator $\mathcal{B}: L^{2}(\Sigma) \rightarrow L^{2}(0, T ; H)$ by

$$
\langle(\mathcal{B} u)(t), \varphi\rangle_{H}=\frac{1}{c_{p}} \int_{\Gamma} u(t, s) \varphi(s) \mathrm{d} \boldsymbol{s} \quad \text { for } \phi \in V, t \in(0, T) \text { a.e. }
$$

then the weak formulation of (2.3) can be expressed in the form (2.2).

It is known [3] that for every $f \in L^{2}(0, T ; H), u \in L^{2}(\mathcal{D})$ and $y_{0} \in V$ there is a unique weak solution $y \in W(0, T) \cap C([0, T] ; V)$ satisfying (2.2). 
Remark 2.2. Let $\hat{y}_{0} \in W(0, T)$ be the unique solution to

$$
\begin{aligned}
\frac{\mathrm{d}}{\mathrm{d} t}\left\langle\hat{y}_{0}(t), \varphi\right\rangle_{H}+a\left(\hat{y}_{0}(t), \varphi\right) & =\langle f(t), \varphi\rangle_{H} & & \text { f.a.a. } t \in[0, T], \forall \varphi \in V, \\
\left\langle\hat{y}_{0}(0), \varphi\right\rangle_{H} & =\left\langle y_{0}, \varphi\right\rangle_{H} & & \forall \varphi \in V .
\end{aligned}
$$

Moreover, we introduce the linear and bounded operator $\mathcal{S}: L^{2}(\mathcal{D}) \rightarrow$ $W(0, T)$ as follows: $\tilde{y}=\mathcal{S} u \in W(0, T)$ is the unique solution to

$$
\begin{aligned}
\frac{\mathrm{d}}{\mathrm{d} t}\langle\tilde{y}(t), \varphi\rangle_{H}+a(\tilde{y}(t), \varphi) & =\langle(\mathcal{B} u)(t), \varphi\rangle_{H} & & \text { f.a.a. } t \in[0, T], \forall \varphi \in V, \\
\langle\tilde{y}(0), \varphi\rangle_{H} & =0 & & \forall \varphi \in V .
\end{aligned}
$$

Then, $y=\hat{y}_{0}+\mathcal{S} u$ is the weak solution to $(2.2)$.

Next we introduce the cost functional $J: W(0, T) \times L^{2}(\mathcal{D}) \rightarrow \mathbb{R}$ by

$$
J(y, u)=\frac{1}{2}\left\|y(T)-y_{d}\right\|_{H}^{2}+\frac{\gamma}{2}\|u\|_{L^{2}(\mathcal{D})}^{2},
$$

where $y_{d} \in H$ holds. Furthermore, $\gamma>0$ is a regularization parameter.

Remark 2.3. We continue Example 2.3. Then, (2.4) yields the cost functional

$$
J(y, u)=\frac{1}{2} \int_{\Omega}\left|y(T)-y_{d}\right|^{2} \mathrm{~d} \boldsymbol{x}+\frac{\gamma}{2} \int_{0}^{T} \int_{\Gamma}|u(t, \boldsymbol{s})|^{2} \mathrm{~d} \boldsymbol{s} \mathrm{d} t
$$

for $(y, u) \in W(0, T) \times L^{2}(\Sigma)$.

The optimal control problem is given by

$$
\min J(y, u) \quad \text { subject to (s.t.) } \quad(y, u) \in W(0, T) \times U_{\text {ad }} \text { solves }(2.2) \text {. }
$$

Applying standard arguments [16] one can prove that there exists a unique optimal solution $\bar{x}=(\bar{y}, \bar{u})$ to $(\mathbf{P})$. Throughout this paper, a bar indicates optimality.

\subsection{First-order optimality conditions}

First-order necessary optimality conditions for our parabolic optimal control problem are well known. We briefly recall them here. Suppose that $\bar{x}=(\bar{y}, \bar{u})$ is the optimal solution to $(\mathbf{P})$. Then there exists a unique Lagrange-multiplier $\bar{p} \in W(0, T)$ satisfying together with $\bar{x}$ the first-order necessary optimality conditions, which consist of the state equations (2.2), the adjoint equations

$$
\begin{array}{ll}
-\frac{\mathrm{d}}{\mathrm{d} t}\langle\bar{p}(t), \varphi\rangle_{H}+a(\bar{p}(t), \varphi)=0 & \text { f.a.a. } t \in[0, T], \forall \varphi \in V, \\
\langle\bar{p}(T), \varphi\rangle_{H}=\left\langle y_{d}-\bar{y}(T), \varphi\right\rangle_{H} & \forall \varphi \in V
\end{array}
$$

and of the variational inequality

$$
\left\langle\gamma \bar{u}-\mathcal{B}^{\star} \bar{p}, u-\bar{u}\right\rangle_{L^{2}(\mathcal{D})} \geq 0 \quad \forall u \in U_{\text {ad }}
$$

Here, the linear and bounded operator $\mathcal{B}^{\star}: L^{2}(0, T ; H) \rightarrow L^{2}(\mathcal{D})^{\prime} \sim L^{2}(\mathcal{D})$ stands for the dual operator of $\mathcal{B}$ satisfying

$$
\langle\mathcal{B} u, \varphi\rangle_{L^{2}(0, T ; H)}=\left\langle u, \mathcal{B}^{\star} \varphi\right\rangle_{L^{2}(\mathcal{D})}=\left\langle\mathcal{B}^{\star} \varphi, u\right\rangle_{L^{2}(\mathcal{D})}
$$

for all $(u, \varphi) \in L^{2}(\mathcal{D}) \times L^{2}(0, T ; H)$. 
Remark 2.4. We continue the discussion of Example 2.1 and Remark 2.3. The adjoint equations (2.5) are given by

$$
\begin{aligned}
-c_{p} \bar{p}_{t}(t, \boldsymbol{x})-\Delta \bar{p}(t, \boldsymbol{x}) & =0 & & \text { f.a.a. }(t, \boldsymbol{x}) \in Q, \\
\frac{\partial \bar{p}}{\partial n}(t, \boldsymbol{s})+q \bar{p}(t, \boldsymbol{s}) & =0 & & \text { f.a.a. }(t, \boldsymbol{s}) \in \Sigma, \\
\bar{p}(T, \boldsymbol{x}) & =y_{d}(\boldsymbol{x})-\bar{y}(T, \boldsymbol{x}) & & \text { f.a.a. } \boldsymbol{x} \in \Omega .
\end{aligned}
$$

Moreover, the variational inequality (2.6) has the form

$$
\int_{0}^{T} \int_{\Gamma}(\gamma \bar{u}(t, s)-\bar{p}(t, s))(u(t, s)-\bar{u}(t, s)) \mathrm{d} s \mathrm{~d} t \geq 0 \quad \text { for all } u \in U_{\text {ad }}
$$

and $\mathcal{B}^{\star} \bar{p}$ is given by $\left(\mathcal{B}^{\star} \bar{p}\right)(t)=\left(\tau_{\Gamma} \bar{p}\right)(t)$ f.a.a. $t \in[0, T]$, where $\tau_{\Gamma}: V \rightarrow L^{2}(\Gamma)$ denotes the common trace operator.

\section{The POD Galerkin discretization}

Problem $(\mathbf{P})$ is an infinite-dimensional problem. Therefore, we have to discretize $(\mathbf{P})$ for its numerical solution. For the discretization of the spatial variable we apply a POD Galerkin approximation, which is discussed now. Let $X$ denote either the space $H$ or the space $V$.

\subsection{The POD method}

Let an arbitrary $u \in U$ be chosen such that the corresponding state variable $y=\hat{y}_{0}+\mathcal{S} u \in W(0, T)$ belongs to $C([0, T] ; V) \hookrightarrow C([0, T] ; X)$. Then,

$$
\mathcal{V}=\operatorname{span}\{y(t) \mid t \in[0, T]\} \subseteq V \subset X .
$$

If $y_{0} \neq 0$ holds, then $\operatorname{span}\left\{y_{0}\right\} \subset \mathcal{V}$ and $d=\operatorname{dim} \mathcal{V} \in[1, \infty]$, but $\mathcal{V}$ may have infinite dimension. We define a bounded linear operator $\mathcal{Y}: L^{2}(0, T) \rightarrow X$ by

$$
\mathcal{Y} \varphi=\int_{0}^{T} \varphi(t) y(t) \mathrm{d} t \quad \text { for } \varphi \in L^{2}(0, T) .
$$

Its Hilbert space adjoint $\mathcal{Y}^{\star}: X \rightarrow L^{2}(0, T)$ satisfying

$$
\langle\mathcal{Y} \varphi, z\rangle_{X}=\left\langle\varphi, \mathcal{Y}^{\star} z\right\rangle_{L^{2}(0, T)} \quad \text { for }(\varphi, z) \in L^{2}(0, T) \times X
$$

is given by $\left(\mathcal{Y}^{\star} z\right)(t)=\langle z, y(t)\rangle_{X}$ for $z \in X$ and f.a.a. $t \in[0, T]$. The bounded linear operator $\mathcal{R}=\mathcal{Y}^{\star} \mathcal{Y}^{\star}: X \rightarrow \mathcal{V} \subset X$ has the form

$$
\mathcal{R} z=\int_{0}^{T}\langle z, y(t)\rangle_{X} y(t) \mathrm{d} t \quad \text { for } z \in X
$$

Moreover, let $\mathcal{K}=\mathcal{Y}^{\star} \mathcal{Y}: L^{2}(0, T) \rightarrow L^{2}(0, T)$ be defined by

$$
(\mathcal{K} \varphi)(t)=\int_{0}^{T}\langle y(\tau), y(t)\rangle_{X} \varphi(\tau) \mathrm{d} \tau \quad \text { for } \varphi \in L^{2}(0, T) .
$$


It is known [11, Section 3] that the operator $\mathcal{R}$ is self-adjoint, compact and non-negative. Thus, that there exists a complete orthonormal basis $\left\{\psi_{i}\right\}_{i=1}^{d}$ for $\mathcal{V}=\operatorname{range}(\mathcal{R}) \subseteq V$ and a sequence $\left\{\lambda_{i}\right\}_{i=1}^{d}$ of real numbers such that

$$
\mathcal{R} \psi_{i}=\lambda_{i} \psi_{i} \text { for } i=1, \ldots, d \quad \text { and } \quad \lambda_{1} \geq \lambda_{2} \geq \ldots \geq \lambda_{d} \geq 0 \text {. }
$$

Remark 3.1. 1) The linear, bounded, compact and self-adjoint operator $\mathcal{K}$ has the same eigenvalues $\left\{\lambda_{i}\right\}_{i=1}^{d}$ as the operator $\mathcal{R}$. For all $\lambda_{i}>0$ the corresponding eigenfunctions of $\mathcal{K}$ are given by

$$
v_{i}(t)=\frac{1}{\sqrt{\lambda_{i}}}\left(\mathcal{Y}^{*} \psi_{i}\right)(t)=\frac{1}{\sqrt{\lambda_{i}}}\left\langle\psi_{i}, y(t)\right\rangle_{X} \text { f.a.a. } t \in[0, T] \text { and } 1 \leq i \leq \ell .
$$

2) Notice that $\mathcal{V} \subset V$ implies $\psi_{i} \in V$ for $1 \leq i \leq \ell$.

For $\ell \leq d$ the eigenvalues and eigenfunctions of $\mathcal{R}$ solve

$$
\min \int_{0}^{T}\left\|y(t)-\sum_{i=1}^{\ell}\left\langle y(t), \psi_{i}\right\rangle_{X} \psi_{i}\right\|_{X}^{2} \mathrm{~d} t \text { s.t. }\left\langle\psi_{j}, \psi_{i}\right\rangle_{X}=\delta_{i j}, 1 \leq i, j \leq \ell .
$$

In particular,

$$
\int_{0}^{T}\left\|y(t)-\sum_{i=1}^{\ell}\left\langle y(t), \psi_{i}\right\rangle_{X} \psi_{i}\right\|_{X}^{2} \mathrm{~d} t=\sum_{i=\ell+1}^{d} \lambda_{i}
$$

\subsection{The discrete POD method}

In real computations, we do not have the whole trajectory $y(t)$ for all $t \in$ $[0, T]$. For that purpose let $0 \leq t_{1}<t_{2}<\ldots<t_{n} \leq T$ be a given time grid and let $y_{j}^{h} \approx y\left(t_{j}\right)$ denote approximations in a finite-dimensional space $X^{h} \subset X$ for $y$ at time instance $t_{j}, j=1, \ldots, n$. We set $\mathcal{V}^{n}=\operatorname{span}\left\{y_{1}^{h}, \ldots, y_{n}^{h}\right\}$ with $d^{n}=\operatorname{dim} \mathcal{V}^{n} \leq n$. Then, for given $\ell \leq n$ we consider the problem

$$
\min \sum_{j=1}^{n} \alpha_{j}\left\|y_{j}^{h}-\sum_{i=1}^{\ell}\left\langle y_{j}^{h}, \psi_{i}^{n}\right\rangle_{X} \psi_{i}^{n}\right\|_{X}^{2} \text { s.t. }\left\langle\psi_{i}^{n}, \psi_{j}^{n}\right\rangle_{X}=\delta_{i j}, 1 \leq i, j \leq \ell
$$

instead of (3.4). In (3.5), the $\alpha_{j}$ 's stand for the trapezoidal weights

$$
\alpha_{1}=\frac{t_{2}-t_{1}}{2}, \quad \alpha_{j}=\frac{t_{j+1}-t_{j-1}}{2} \text { for } 2 \leq j \leq n-1, \quad \alpha_{n}=\frac{t_{n}-t_{n-1}}{2} .
$$

The solution to (3.5) is given by the solution to the eigenvalue problem

$$
\mathcal{R}^{n} \psi_{i}^{n}=\sum_{j=1}^{n} \alpha_{j}\left\langle y_{j}^{h}, \psi_{i}^{n}\right\rangle_{X} y_{j}^{h}=\lambda_{i}^{n} \psi_{i}^{n}, \quad i=1, \ldots, \ell,
$$

where $\mathcal{R}^{n}: X^{h} \rightarrow \mathcal{V}^{n} \subset V$ is a linear, bounded, compact, self-adjoint and non-negative operator. Thus, there are an orthonormal set $\left\{\psi_{i}^{n}\right\}_{i=1}^{d^{n}}$ of eigenfunctions and corresponding non-negative eigenvalues $\left\{\lambda_{i}^{n}\right\}_{i=1}^{d^{n}}$ satisfying

$$
\mathcal{R}^{n} \psi_{i}^{n}=\lambda_{i}^{n} \psi_{i}^{n}, \quad \lambda_{1}^{n} \geq \lambda_{2}^{n} \geq \ldots \geq \lambda_{d^{n}}^{n}>0 .
$$

We refer to [14] for the relationship between (3.3) and (3.6). 
Remark 3.2. Let $X^{h}$ be given by the subset $\operatorname{span}\left\{\varphi_{1}, \ldots, \varphi_{m}\right\} \subset X$, where the $\varphi_{i}$ 's are assumed to be linearly independent in $X$. Then we have

$$
y_{j}^{h}(\boldsymbol{x})=\sum_{i=1}^{m} Y_{i j} \varphi_{i}(\boldsymbol{x}) \in X^{h} \quad \text { for } \boldsymbol{x} \in \Omega \text { and } j=1, \ldots, m
$$

with real coefficients $Y_{i j}$. In this case the POD basis functions are given by

$$
\psi_{j}^{n}(\boldsymbol{x})=\sum_{i=1}^{m} \Psi_{i j} \varphi_{i}(\boldsymbol{x}) \in X^{h} \quad \text { for } \boldsymbol{x} \in \Omega \text { and } j=1, \ldots, \ell
$$

with real coefficients $\Psi_{i j}$. Then we have to determine the coefficient matrix $\Psi=\left(\left(\Psi_{i j}\right)\right) \in \mathbb{R}^{m \times \ell}$. For that purpose we define $Y=\left(\left(Y_{i j}\right)\right) \in \mathbb{R}^{m \times n}$ and $W=\left(\left(\left\langle\varphi_{j}, \varphi_{i}\right\rangle_{X}\right)\right) \in \mathbb{R}^{m \times m}$. Moreover, we define the diagonal matrix $D=\operatorname{diag}\left(\alpha_{1}, \ldots, \alpha_{n}\right) \in \mathbb{R}^{n \times n}$ and set $\bar{Y}=W^{1 / 2} Y D^{1 / 2} \in \mathbb{R}^{m \times n}$. Then $\Psi=\left[u_{1}, \ldots, u_{n}\right]$ can be computed as follows (see, e.g., [23, Section 1.3])

1) Solve the $m \times m$ eigenvalue problem

$$
\bar{Y} \bar{Y}^{T} \mathfrak{u}_{i}=\lambda_{i} \mathfrak{u}_{i}, 1 \leq i \leq \ell, \quad \text { with } \quad \mathfrak{u}_{i}^{T} \mathfrak{u}_{j}=\delta_{i j}, 1 \leq i, j \leq \ell,
$$

for the largest eigenvalues $\lambda_{1} \geq \lambda_{2} \geq \ldots \geq \lambda_{\ell}>0$ and compute $u_{i}=$ $W^{-1 / 2} \mathfrak{u}_{i}$. Since $\bar{Y} \bar{Y}^{T}=W^{1 / 2} Y D Y^{\bar{T}} W^{1 / 2}$ holds, this variant is often numerically expensive, especially for $m \gg n$.

2) Solve the $n \times n$ eigenvalue problem

$$
\bar{Y}^{T} \bar{Y} \mathfrak{v}_{i}=\lambda_{i} \mathfrak{v}_{i}, 1 \leq i \leq \ell, \quad \text { with } \quad \mathfrak{v}_{i}^{T} \mathfrak{v}_{j}=\delta_{i j}, 1 \leq i, j \leq \ell,
$$

for the largest eigenvalues $\lambda_{1} \geq \lambda_{2} \geq \ldots \geq \lambda_{\ell}>0$ and set $u_{i}=$ $Y D^{1 / 2} \mathfrak{v}_{i} / \sqrt{\lambda_{i}}$. To solve (3.7) we apply the MATLAB routine eigs and call this variant 'eigs' in Section 4 . Note that $\bar{Y}^{T} \bar{Y}=D^{1 / 2} Y^{T} W Y D^{1 / 2}$ holds and $D$ is a diagonal matrix. Since we do not have to compute $W^{1 / 2}$, this variant is very attractive for $n \leq m$. We will apply this approach in our numerical experiments.

3) Compute the singular value decomposition (SVD) of $\bar{Y}$, i.e., determine orthonormal vectors $\left\{\mathfrak{u}_{i}\right\}_{i=1}^{\ell}$ in $\mathbb{R}^{m}$ and $\left\{\mathfrak{v}_{i}\right\}_{i=1}^{\ell}$ in $\mathbb{R}^{n}$ associated with the largest singular values $\sigma_{1} \geq \sigma_{2} \geq \ldots \geq \sigma_{\ell}>0$ satisfying

$$
\bar{Y} \mathfrak{v}_{i}=\sigma_{i} \mathfrak{u}_{i}, \quad \bar{Y}^{T} \mathfrak{u}_{i}=\sigma_{i} \mathfrak{v}_{i}, \quad 1 \leq i \leq \ell .
$$

(see, e.g., [18]). It follows that $\lambda_{i}=\sigma_{i}^{2}$ and $u_{i}=W^{-1 / 2} \mathfrak{u}_{i}$. Since this variant is based on the SVD, we call this variant 'SVD' in Section 4. Although the computation of $W^{1 / 2}$ is costly, the SVD is known to be more stable. This is due to the fact that the products of $\bar{Y}$ and $\bar{Y}^{T}$ doubles the condition number of the problem compared to the SVD. $\diamond$

\subsection{POD Galerkin approximation for $(\mathbf{P})$}

Let $y=\hat{y}_{0}+\mathcal{S} u$ be the state associated with some control $u \in U$, and let $\mathcal{V}$ be given as in (3.1). We fix $\ell$ with $\ell \leq d$ and compute the first $\ell$ POD basis functions $\psi_{1}, \ldots, \psi_{\ell} \in V$ by solving either $\mathcal{R} \psi_{i}=\lambda_{i} \psi_{i}$ or $\mathcal{K} v_{i}=\lambda v_{i}$ for $i=1, \ldots, \ell($ see Remark 3.1). Then we define

$$
V^{\ell}=\operatorname{span}\left\{\psi_{1}, \ldots, \psi_{\ell}\right\} \subset V \text {. }
$$


Endowed with the topology in $V$ it follows that $V^{\ell}$ is a Hilbert space. The POD Galerkin scheme for the state equation (2.2) leads to the following linear problem: determine a function $y^{\ell}(t) \in V^{\ell}$ such that

$$
\begin{aligned}
\frac{\mathrm{d}}{\mathrm{d} t}\left\langle y^{\ell}(t), \psi\right\rangle_{H}+a\left(y^{\ell}(t), \psi\right) & =\langle(f+\mathcal{B} u)(t), \psi\rangle_{H} \\
& \text { f.a.a. } t \in[0, T], \forall \psi \in V^{\ell}, \\
\left\langle y^{\ell}(0), \psi\right\rangle_{H} & =\left\langle y_{0}, \psi\right\rangle_{H} \quad \forall \psi \in V^{\ell} .
\end{aligned}
$$

For every $f \in L^{2}(0, T ; H), u \in L^{2}(\mathcal{D}), y_{0} \in V$ and for every $\ell \in \mathbb{N}$ problem (3.8) admits a unique solution $y^{\ell} \in H^{1}\left(0, T ; V^{\ell}\right)$; see [10, Proposition 3.4]. From $V^{\ell} \hookrightarrow V$ it follows that $y^{\ell} \in W(0, T)$ holds.

The POD Galerkin approximation for $(\mathbf{P})$ is given by problem

$$
\min J\left(y^{\ell}, u\right) \quad \text { s.t. } \quad\left(y^{\ell}, u\right) \in H^{1}\left(0, T ; V^{\ell}\right) \times U_{\text {ad }} \text { solves }(3.8) .
$$

Problem $\left(\mathbf{P}^{\ell}\right)$ admits a unique optimal solution $\bar{x}^{\ell}=\left(\bar{y}^{\ell}, \bar{u}^{\ell}\right)$ that is interpreted as a suboptimal solution to $(\mathbf{P})$. First-order necessary optimality conditions for $\left(\mathbf{P}^{\ell}\right)$ are given by the state equation (3.8) with $u=\bar{u}^{\ell}$, the adjoint equation

$$
\begin{aligned}
-\frac{\mathrm{d}}{\mathrm{d} t}\left\langle\bar{p}^{\ell}(t), \psi\right\rangle_{H}+a\left(\bar{p}^{\ell}(t), \psi\right) & =0 \quad \text { f.a.a. } t \in[0, T], \forall \psi \in V^{\ell}, \\
\left\langle\bar{p}^{\ell}(T), \psi\right\rangle_{H} & =\left\langle y_{d}-\bar{y}^{\ell}(T), \psi\right\rangle_{H} \quad \forall \psi \in V^{\ell} .
\end{aligned}
$$

and the variational inequality

$$
\left\langle\gamma \bar{u}^{\ell}-\mathcal{B}^{\star} \bar{p}^{\ell}, u-\bar{u}^{\ell}\right\rangle_{L^{2}(\mathcal{D})} \geq 0 \quad \text { for all } u \in U_{\text {ad }}
$$

To solve $\left(\mathbf{P}^{\ell}\right)$ we apply a primal-dual active set strategy, which converges locally superlinearly [7]. Its mesh-independence is proved in [8, 9].

\subsection{A-posteriori error estimate for the POD approximation}

In this subsection we present the a-posteriori error estimate for the control variable. The result is taken from [22, Theorem 4.11].

Theorem 3.3. Suppose that $(\bar{y}, \bar{u})$ is the solution to $(\mathbf{P})$. For an arbitrary $\ell \leq d$ let $\left(\bar{y}^{\ell}, \bar{u}^{\ell}\right)$ be the optimal solution to $\left(\mathbf{P}^{\ell}\right)$. Let $\tilde{y}=\hat{y}_{0}+\mathcal{S} \bar{u}^{\ell}$ and $\tilde{p}=\tilde{p}\left(\bar{u}^{\ell}\right)$ be the solution to the associated adjoint equation

$$
\begin{aligned}
-\frac{\mathrm{d}}{\mathrm{d} t}\langle\tilde{p}(t), \varphi\rangle_{H}+a(\tilde{p}(t), \varphi) & =0, & & t \in[0, T], \forall \varphi \in V, \\
\langle\tilde{p}(T), \varphi\rangle_{H} & =\left\langle y_{d}-\tilde{y}(T), \psi\right\rangle_{H} & & \forall \varphi \in V .
\end{aligned}
$$

Define the residual function $\zeta^{\ell} \in L^{2}(\mathcal{D})$ by

$$
\zeta^{\ell}(s)= \begin{cases}{\left[\left(\gamma \bar{u}^{\ell}-\mathcal{B}^{\star} \tilde{p}\right)(s)\right]_{-}} & \text {on } \mathcal{A}_{-}^{\ell}=\left\{s \in \mathcal{D} \mid \bar{u}^{\ell}(s)=u_{a}(s)\right\}, \\ {\left[\left(\gamma \bar{u}^{\ell}-\mathcal{B}^{\star} \tilde{p}\right)(s)\right]_{+}} & \text {on } \mathcal{A}_{+}^{\ell}=\left\{s \in \mathcal{D} \mid \bar{u}^{\ell}(s)=u_{b}(s)\right\} \\ -\left(\gamma \bar{u}^{\ell}-\mathcal{B}^{\star} \tilde{p}\right)(s) & \text { on } \mathcal{J}^{\ell}=\mathcal{D} \backslash\left(\mathcal{A}_{-}^{\ell} \cup \mathcal{A}_{+}^{\ell}\right)\end{cases}
$$


with $[r]_{-}=-\min (0, r)$ and $[r]_{+}=\max (0, r)$. Then

$$
\left\|\bar{u}-\bar{u}^{\ell}\right\|_{L^{2}(\mathcal{D})} \leq \frac{1}{\gamma}\left\|\zeta^{\ell}\right\|_{L^{2}(\mathcal{D})} .
$$

Remark 3.4. 1) Notice that $\tilde{y}$ and $\tilde{p}$ must be taken as the solutions to the (full) state and adjoint equation, respectively, not of their POD-approximations.

2) In [22] sufficient conditions are presented that $\lim _{\ell \rightarrow \infty}\left\|\zeta^{\ell}\right\|_{L^{2}(\mathcal{D})}=0$. Thus, $\left\|\zeta^{\ell}\right\|_{L^{2}(\mathcal{D})}$ can be expected smaller than any $\varepsilon>0$ provided that $\ell$ is taken sufficiently large. Motivated by this result, we set up Algorithm 1.

3) Notice that the presented error estimate holds for time-variant, linearquadratic optimal control problems. For recent extension to nonlinear problems we refer to [13] and to [12, 20], where the presented error estimate is utilized in a multilevel SQP algorithm.

4) To improve the approximation quality of the POD basis, we can combine the a-posteriori analysis with basis update strategies; see [24].

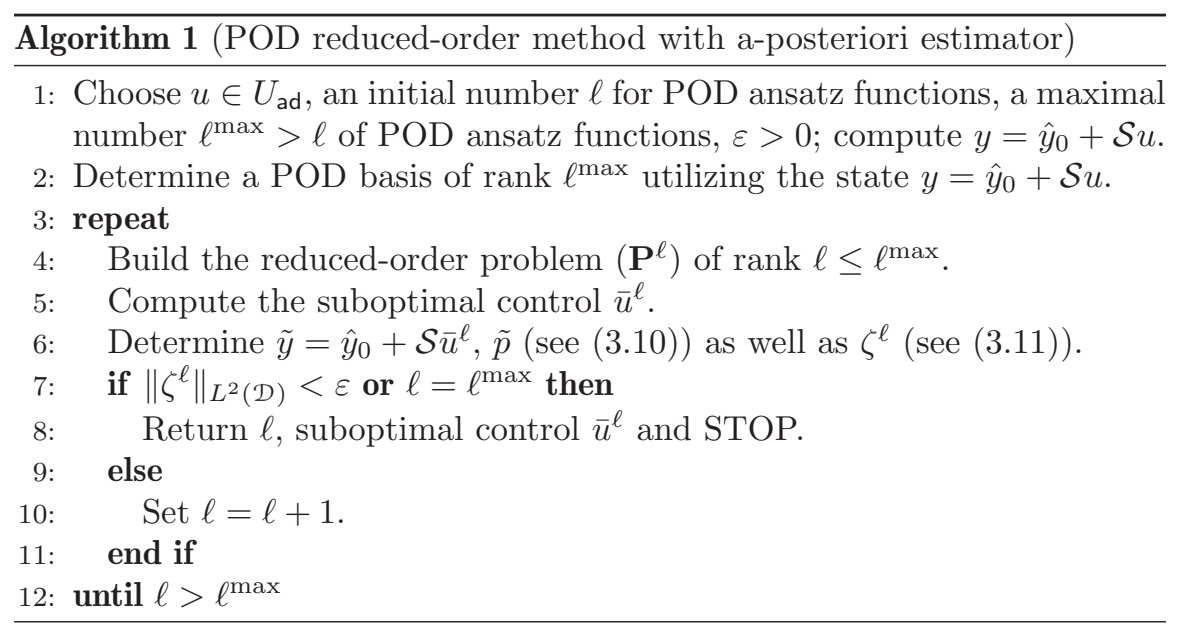

\section{Numerical experiments}

This chapter is devoted to numerical test examples. First, we turn to the numerical solution of a given parabolic PDE. We pursue the two different Galerkin approaches, namely the finite element (FE) Galerkin method and the POD Galerkin technique, and compare the results in order to see some different implementation choices that can be taken, to become a sense of how good these approximate solutions are and to point out some advantages and drawbacks of the considered methods. From solving one linear parabolic equation we move on to applying the POD-Galerkin ansatz to $(\mathbf{P})$. Hereby, 
we especially focus on testing the accuracy and efficiency of the POD aposteriori estimator reviewed in Section 3.4. For the implementation we use the MATLAB software package (R2010a). In all examples we choose the spatial domain is $\Omega=(0,1) \times(0,1) \subset \mathbb{R}^{2}$. The time interval of consideration will always be $[0, T]=[0,1]$. The time integration is carried out by the implicit Euler method with an equidistant time grid $0=t_{0}<t_{1}<\ldots<t_{n}=T$, where $t_{i}=i \Delta t$ and $\Delta t=1 / n$.

Run 4.1 (Heat equation). In (2.3) we choose the data $y_{0} \equiv 0, c_{p}=1, q=0$ and $\tilde{f}(t, \boldsymbol{x})=\cos \left(2 \pi x_{1}\right) \cos \left(2 \pi x_{2}\right)\left(1+8 \pi^{2} t\right),(t, \boldsymbol{x}) \in Q$ and $\boldsymbol{x}=\left(x_{1}, x_{2}\right)$. Then, the exact solution is $y_{e x}(t, \boldsymbol{x})=t \cos \left(2 \pi x_{1}\right) \cos \left(2 \pi x_{2}\right)$ for $(t, \boldsymbol{x}) \in Q$. For the FE method we choose piecewise quadratic elements resulting in $m=$ 665 spatial degrees of freedom. The time increment was chosen to be $\Delta t=$ 0.01 and we have $n=101$. Notice that the discretization error with respect to the spatial and the time variable is of the same size $\mathcal{O}(\Delta t)$. To compute the POD basis we compare the variants 'eigs' and 'SVD'; see Remark 3.2. Choosing $X=H$ in Section 3.1 the rapid decays of the normalized eigenvalues

$$
\bar{\lambda}_{i}=\frac{\lambda_{i}}{\sum_{j=1}^{n} \lambda_{j}}=\frac{\lambda_{i}}{\operatorname{trace}\left(\bar{Y}^{T} \bar{Y}\right)}
$$

and normalized squared singular values $\bar{\sigma}_{i}^{2}=\bar{\lambda}_{i}$ are presented in Figure 1. We
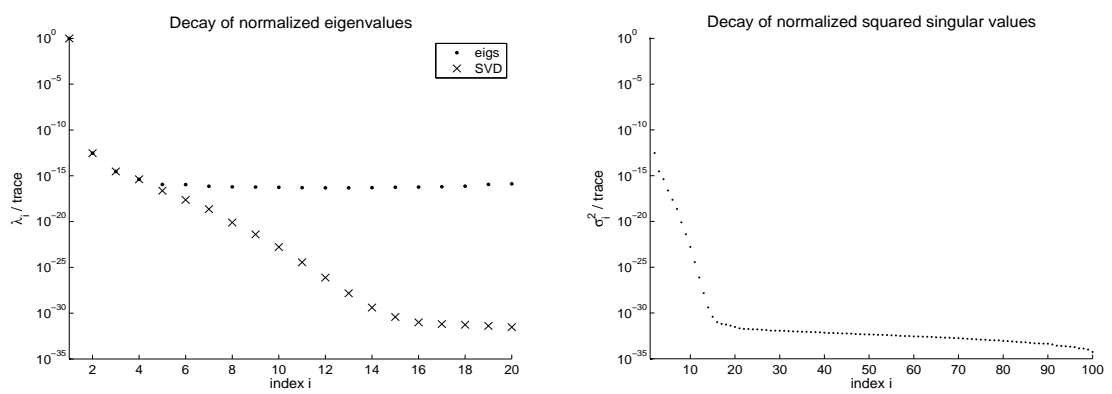

Figure 1. Run 4.1: Decay of $\bar{\lambda}_{i}$ for 'eigs' (left plot) and $\bar{\sigma}_{i}^{2}$ for 'SVD' (right plot) with $X=H$.

observe that in the beginning the eigenvalues are equal in deed, whereas the eigenvalues computed with the SVD keep decreasing when the eigenvalues for 'eigs' stagnate at the order of machine precision. The difference between the 'eigs' and the 'SVD' variant shows already for small $\ell$ in this example due to the extremely rapid decay of eigenvalues, see Table 1, where

$$
\varepsilon_{\mathrm{abs}}^{y}(\ell)=\sum_{j=1}^{n} \alpha_{j}\left\|y^{h}\left(t_{j}\right)-y^{\ell}\left(t_{j}\right)\right\|_{H}, \quad \mathcal{E}_{\mathrm{rel}}^{y}(\ell)=\sum_{j=1}^{n} \alpha_{j} \frac{\left\|y^{h}\left(t_{j}\right)-y^{\ell}\left(t_{j}\right)\right\|_{H}}{\left\|y^{h}\left(t_{j}\right)\right\|_{H}}
$$

stands for absolute and relative error between FE and POD solution. In fact, the solution space of the PDE is one-dimensional, since the exact solution at time $t$ is given by a multiple of $\cos \left(2 \pi x_{1}\right) \cos \left(2 \pi x_{2}\right)$ by the factor $t$. This 


\begin{tabular}{|c|c|c|c|c|c|c|}
\hline \multirow[b]{2}{*}{$\ell$} & \multicolumn{3}{|c|}{ Variant 'eigs' } & \multicolumn{3}{|c|}{ Variant 'SVD' } \\
\hline & $\mathcal{E}_{\text {abs }}^{y}(\ell)$ & $\mathcal{E}_{\mathrm{rel}}^{y}(\ell)$ & $\lambda_{\ell}$ & $\mathcal{E}_{\text {abs }}^{y}(\ell)$ & $\mathcal{E}_{\mathrm{rel}}^{y}(\ell)$ & $\lambda_{\ell}$ \\
\hline 1 & $1.3 \cdot 10^{-07}$ & $2.1 \cdot 10^{-06}$ & $8.5 \cdot 10^{-00}$ & $1.3 \cdot 10^{-07}$ & $2.1 \cdot 10^{-06}$ & $8.5 \cdot 10^{-00}$ \\
\hline 2 & $1.4 \cdot 10^{-08}$ & $5.8 \cdot 10^{-07}$ & $2.5 \cdot 10^{-12}$ & $1.4 \cdot 10^{-08}$ & $5.8 \cdot 10^{-07}$ & $2.5 \cdot 10^{-12}$ \\
\hline 5 & $4.3 \cdot 10^{-10}$ & $1.1 \cdot 10^{-08}$ & $1.1 \cdot 10^{-15}$ & $4.3 \cdot 10^{-10}$ & $1.1 \cdot 10^{-08}$ & $2.0 \cdot 10^{-16}$ \\
\hline 10 & $3.2 \cdot 10^{-13}$ & $5.4 \cdot 10^{-12}$ & $4.6 \cdot 10^{-16}$ & $1.6 \cdot 10^{-13}$ & $2.7 \cdot 10^{-12}$ & $1.4 \cdot 10^{-22}$ \\
\hline 11 & $6.0 \cdot 10^{-13}$ & $7.8 \cdot 10^{-12}$ & $4.4 \cdot 10^{-16}$ & $2.2 \cdot 10^{-14}$ & $3.4 \cdot 10^{-13}$ & $3.1 \cdot 10^{-24}$ \\
\hline 14 & $7.7 \cdot 10^{-12}$ & $3.1 \cdot 10^{-11}$ & $4.4 \cdot 10^{-16}$ & $5.2 \cdot 10^{-16}$ & $2.5 \cdot 10^{-15}$ & $3.5 \cdot 10^{-29}$ \\
\hline 15 & $9.9 \cdot 10^{-12}$ & $4.0 \cdot 10^{-11}$ & $4.6 \cdot 10^{-16}$ & $5.5 \cdot 10^{-16}$ & $2.5 \cdot 10^{-15}$ & $3.2 \cdot 10^{-30}$ \\
\hline
\end{tabular}

TABle 1. Run 4.1: Absolute \& relative errors and $\lambda_{\ell}$ for different $\ell$ and for 'eigs' and 'SVD' using $X=H$.

behaviour is already captured by one mode/basis function. Due to the inaccuracy of the numerical method for determining the snapshots, the snapshot matrix $Y$ representing the solution space has a rank greater than one. Thus the first POD basis function $\psi_{1}$ is not an exact multiple of $\cos \left(2 \pi x_{1}\right) \cos \left(2 \pi x_{2}\right)$, and the dynamics of the PDE can not be described comprehensively with only one POD basis function. Hence, increasing the rank of the POD basis leads the approximation quality to rise.

The fact that the eigenvalues for 'eigs' increase starting from $\ell=14$ instead of continuing to decrease like for 'SVD' illustrate that the SVD is more stable than the eigenvalue solver. Since the $\bar{Y}^{T} \bar{Y}$ is symmetric, positive semi-definite, it should only have real non-negative eigenvalues. Due to rounding errors, eigenvalues that are nearly of the size of the machine precision (eps $=2.2204 \cdot 10^{-16}$ ) can be mistakenly determined as negative or complex by eigs.m. For SVD the eigenvalues keep on decreasing since they are computed as squares of the obtained singular values. This leads also to a monotone decrease of the quantities $\varepsilon_{\text {abs }}^{y}(\ell)$ and $\varepsilon_{\text {rel }}^{y}(\ell)$ for the SVD, whereas 'eigs' yields a stagnation of the error for $\ell \geq 14$. A related aspect is the $W$-orthonormality of the POD basis vectors. Analytically, we have

$$
\Psi_{\cdot, 1: \ell}^{T} W \Psi_{\cdot, 1: \ell}-I_{\ell} \stackrel{!}{=} 0,
$$

where $I_{\ell}$ denotes the $\ell$ by $\ell$ identity matrix and $\Psi(:, 1: \ell)$ contains the first $\ell$ columns of $\psi$. We estimate the spectral norm $\left\|\Psi_{\cdot, 1: \ell}^{T} W \Psi_{\cdot, 1: \ell}-I_{\ell}\right\|_{2}$ by utilizing the MATLAB routine normest; compare Table 2. We observe that the 'SVD'

\begin{tabular}{r|cc}
\hline & \multicolumn{2}{|c}{$\left\|\Psi_{\cdot, 1: \ell}^{T} W \Psi \cdot, 1: \ell-I_{\ell}\right\|_{2}$} \\
$\ell$ & Variant 'eigs' & Variant 'SVD' \\
\hline 1 & $8.12 \cdot 10^{-16}$ & $2.66 \cdot 10^{-15}$ \\
2 & $3.51 \cdot 10^{-05}$ & $3.98 \cdot 10^{-15}$ \\
5 & $9.95 \cdot 10^{-01}$ & $3.94 \cdot 10^{-15}$ \\
10 & $9.99 \cdot 10^{-01}$ & $1.05 \cdot 10^{-14}$ \\
\hline
\end{tabular}

TABLE 2. Run 4.1: Spectral norm $\left\|\Psi_{\cdot, 1: \ell}^{T} W \Psi \cdot, 1: \ell-I_{\ell}\right\|_{2}$ for differnt $\ell$ and for 'eigs' \& 'SVD' using $X=H$.

approach fullfils the $W$-orthogonality far better than the 'eigs' variant. From 
Tables 1 and 2, we can deduce that especially for higher POD basis rank the SVD is more stable or accurate. However, we should mention that the 'SVD' variant is more costly than the 'eigs' variant, especially if the number of spatial degrees of freedom $m$ is much bigger that the number $n$ of time steps.

Run 4.2 (Unconstrained optimal control). In the context of Example 2.1, Remark 2.3 and Remark 2.4 we choose $\gamma=10^{-2}, c_{p}=10, q=0.01$ and $\tilde{f} \equiv 0$. The initial condition is $y_{0}(\boldsymbol{x})=3-4\left(x_{2}-0.5\right)^{2}$ and the desired state is $y_{d}(\boldsymbol{x})=2+2\left|2 x_{1}-x_{2}\right|$ for $\boldsymbol{x}=\left(x_{1}, x_{2}\right) \in \Omega$. Choosing $u_{a}=-\infty=-u_{b}$ we have $U_{\text {ad }}=L^{2}(\Sigma)$. We make use of the MATLAB PDE toolbox for the spatial discretization with piecewise linear, continuous finite elements $\left(P_{1}\right.$ Elements) with maximal edge length $h_{\max }=0.06$ and thus $N_{F E}=498$ degrees of freedom. For the implicit Euler method we choose the step size $\Delta t=0.004$. The FE optimal control $\bar{u}^{h}$ is presented at all times in Figure 2 . The different tested ROM runs vary in the way the POD basis is determined:
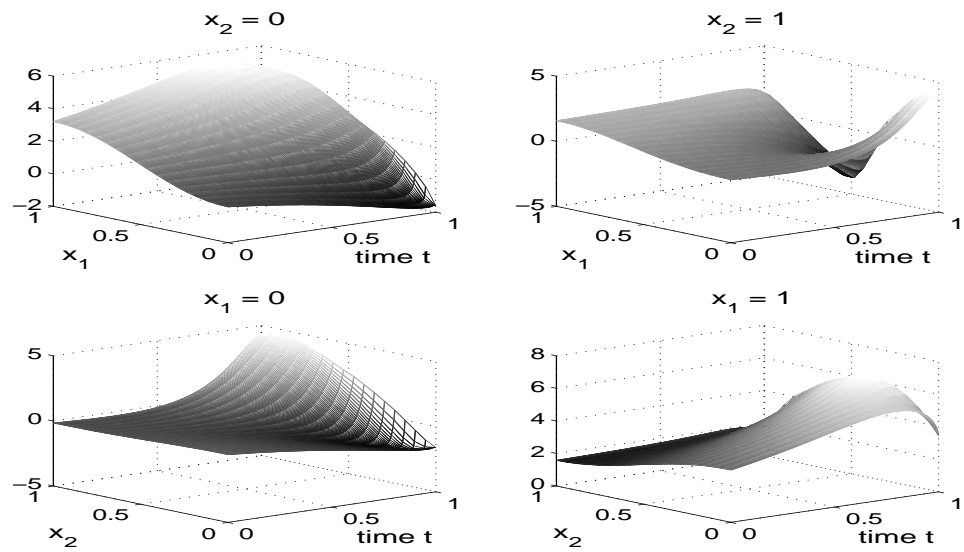

Figure 2. Run 4.2: FE optimal control $\bar{u}^{h}(t, \boldsymbol{x})$ for $\boldsymbol{x}=$ $\left(x_{1}, 0\right) \in \Gamma$ (upper left plot), $\boldsymbol{x}=\left(x_{1}, 1\right) \in \Gamma$ (upper right plot), $\boldsymbol{x}=\left(0, x_{2}\right) \in \Gamma$ (lower left plot), $\boldsymbol{x}=\left(1, x_{2}\right) \in \Gamma$ (lower right plot).

1) To generate the snapshots for the POD method we have to solve (2.3) for a reference control $u=u_{\text {ref. }}$. We consider three different reference controls:

1a) $u_{\text {ref }}^{1}(t, s)=\exp (t)\left(\frac{1}{2}\left|2 x_{1}-x_{2}\right|+\frac{1}{3}\left(\sin \left(\pi x_{2}\right)-1\right)\right)$ for $(t, s) \in \Sigma$, $\boldsymbol{s}=\left(x_{1}, x_{2}\right)$ (see Figure 3$)$;

1b) $u_{\text {ref }}^{2}(t, \boldsymbol{x})=u^{h}(t, \boldsymbol{x})$ for $(t, \boldsymbol{x}) \in \Sigma$ (see Figure 2).

The reference control $u_{\text {ref }}^{1}$ is plotted in Figure 3 .

2) The snapshot ensemble to be represented well by the POD basis can now be taken from the solution $y_{\text {ref }}$ of the state equation with $u=$ 
$u_{\mathrm{ref}}^{i}, i=1,2$, which is called Variant 1 . If we want/need to enrich the approximation space, we also solve the adjoint equation with $y=y_{\text {ref }}$ and then consider a snapshot ensemble consisting of snapshots from both the state and the adjoint equation. This approach is called Variant 2. Let us note that another possibility would be to use two different bases which is not considered in this thesis.

3) For the POD basis computation we choose

3a) 'eigs' includes solving $\bar{Y}^{T} \bar{Y} \mathfrak{v}=\lambda \mathfrak{v}$ with $\bar{Y}=W^{1 / 2} Y D^{1 / 2}$;

3b) 'SVD' involves the singular value decomposition of $\bar{Y}$.
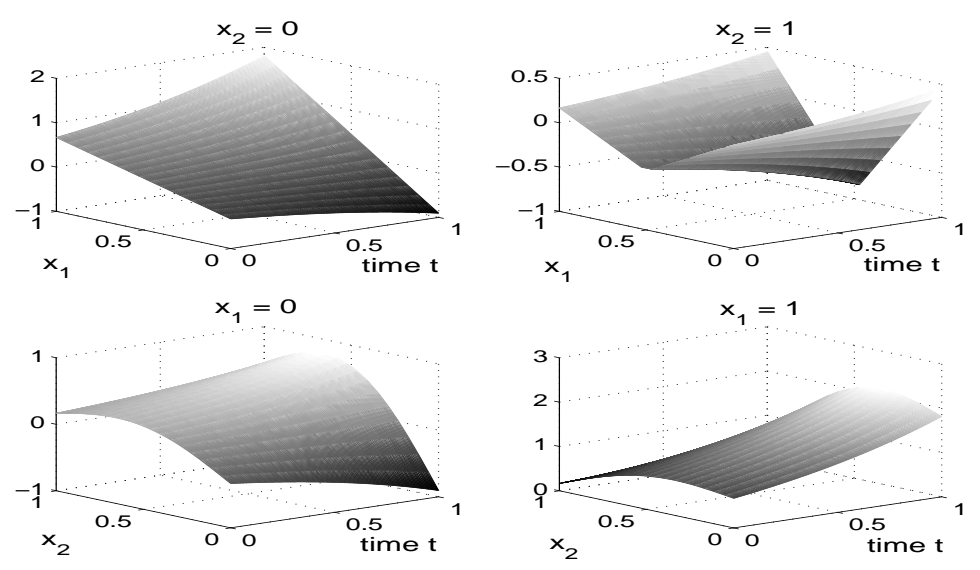

Figure 3. Run 4.2: $u_{\text {ref }}^{1}$ for $\boldsymbol{x}=\left(x_{1}, 0\right) \in \Gamma$ (upper left plot), $\boldsymbol{x}=\left(x_{1}, 1\right) \in \Gamma$ (upper right plot), $\boldsymbol{x}=\left(0, x_{2}\right) \in \Gamma$ (lower left plot), $\boldsymbol{x}=\left(1, x_{2}\right) \in \Gamma$ (lower right plot).

Moreover, the POD basis can be computed for the choices $X=H$ or $X=V$. First, we choose $u_{\text {ref }}^{1}$ for the snapshot generation and use 'eigs' to determine the POD basis based on a snapshot ensemble from both the state and the adjoint equation (Variant 2). In order to get a first idea of how many POD basis functions we should use in the POD-Galerkin ansatz for the state and the adjoint state variable, we look at the decay of the eigenvalues; see Figure 4. Naturally, in the case of the $V$-norm the eigenvalues decay slower than with the discrete $H$-norm. The decay plot shows where the eigenvalues stagnate. Usually, from that number of POD basis functions on, we can not further or significantly improve the approximation errors any more. Theoretically, increasing the POD basis rank leads to a decrease in approximation error values. Nevertheless, we have to pay attention if we use the not so stable method 'eigs' for POD basis computation. The instability can be detected in the right-hand side plot of Figure 4 for $\ell>60$. Note that due to the slower decay of eigenvalues with the $H^{1}$-norm implementations those errors are still decreasing for up to $\ell=70$ POD basis functions. The instability sets in later. 

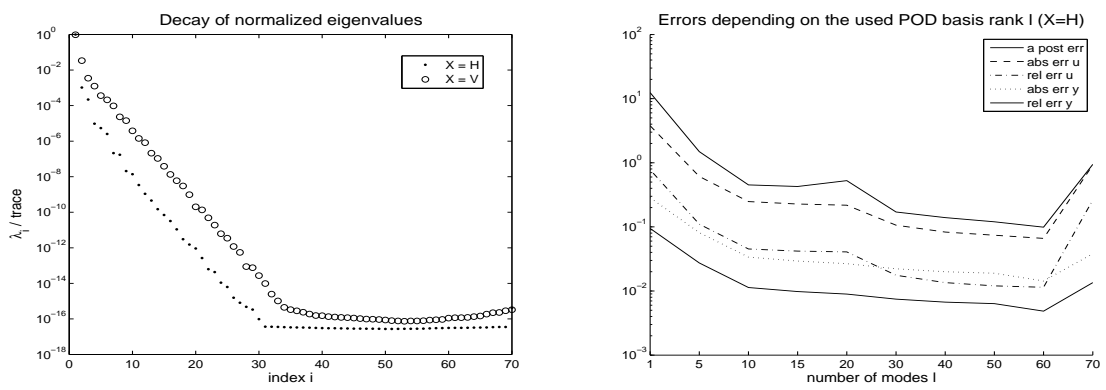

Figure 4. Run 4.2: Decay of $\bar{\lambda}_{i}$ for $X=H$ and $X=V$ (left plot) and decay of the a-posteriori error estimator, the absolute as well as the relative errors using $u_{\text {ref }}^{1}$ and 'eigs'.

Let us define the quantities

$$
\begin{aligned}
\mathcal{E}_{\mathrm{abs}}^{u}(\ell) & =\sum_{j=0}^{n} \alpha_{j}\left\|\bar{u}^{h}\left(t_{j}\right)-\bar{u}^{\ell}\left(t_{j}\right)\right\|_{L^{2}(\Gamma)}, \\
\mathcal{E}_{\mathrm{rel}}^{u}(\ell) & =\sum_{j=0}^{n} \alpha_{j} \frac{\left\|\bar{u}^{h}\left(t_{j}\right)-\bar{u}^{\ell}\left(t_{j}\right)\right\|_{L^{2}(\Gamma)}}{\left\|\bar{u}^{h}\left(t_{j}\right)\right\|_{L^{2}(\Gamma)}}
\end{aligned}
$$

The errors which occurred between the (sub-)optimal controls within the first two ROM runs compared to the FE based approaches are listed in Table 3. The obtained results for the POD suboptimal controls are not satisfying.

\begin{tabular}{r|ccc|ccc}
\hline \multicolumn{1}{c|}{} & $\left\|\zeta^{\ell}\right\| / \gamma$ & $\begin{array}{c}X=H \\
\mathcal{E}_{\text {abs }}^{u}(\ell)\end{array}$ & $\mathcal{E}_{\text {rel }}^{u}(\ell)$ & $\left\|\zeta^{\ell}\right\| / \gamma$ & $\begin{array}{c}X=V \\
\mathcal{E}_{\text {abs }}^{u}(\ell)\end{array}$ & $\mathcal{E}_{\text {rel }}^{u}(\ell)$ \\
\hline 1 & $1.2 \cdot 10^{+1}$ & $3.8 \cdot 10^{-0}$ & $7.8 \cdot 10^{-1}$ & $1.2 \cdot 10^{+1}$ & $3.8 \cdot 10^{-0}$ & $7.8 \cdot 10^{-1}$ \\
5 & $1.5 \cdot 10^{-0}$ & $6.1 \cdot 10^{-1}$ & $1.1 \cdot 10^{-1}$ & $2.4 \cdot 10^{-0}$ & $9.8 \cdot 10^{-1}$ & $1.8 \cdot 10^{-1}$ \\
10 & $4.5 \cdot 10^{-1}$ & $2.5 \cdot 10^{-1}$ & $4.5 \cdot 10^{-2}$ & $4.4 \cdot 10^{-1}$ & $2.5 \cdot 10^{-1}$ & $4.6 \cdot 10^{-2}$ \\
20 & $5.3 \cdot 10^{-1}$ & $2.2 \cdot 10^{-1}$ & $4.1 \cdot 10^{-2}$ & $5.5 \cdot 10^{-1}$ & $2.1 \cdot 10^{-1}$ & $3.9 \cdot 10^{-2}$ \\
30 & $1.7 \cdot 10^{-1}$ & $1.1 \cdot 10^{-1}$ & $1.8 \cdot 10^{-2}$ & $1.7 \cdot 10^{-1}$ & $1.1 \cdot 10^{-1}$ & $1.8 \cdot 10^{-2}$ \\
50 & $1.2 \cdot 10^{-1}$ & $7.4 \cdot 10^{-2}$ & $1.2 \cdot 10^{-2}$ & $1.2 \cdot 10^{-1}$ & $7.5 \cdot 10^{-2}$ & $1.2 \cdot 10^{-2}$ \\
60 & $9.9 \cdot 10^{-2}$ & $6.6 \cdot 10^{-2}$ & $1.1 \cdot 10^{-2}$ & $5.8 \cdot 10^{-2}$ & $4.2 \cdot 10^{-2}$ & $6.8 \cdot 10^{-3}$ \\
70 & $9.4 \cdot 10^{-1}$ & $9.3 \cdot 10^{-1}$ & $2.6 \cdot 10^{-1}$ & $5.2 \cdot 10^{-2}$ & $3.9 \cdot 10^{-2}$ & $6.2 \cdot 10^{-3}$ \\
\hline
\end{tabular}

TABLE 3. Run 4.2: A-posteriori estimator, absolute and relative errors in the control variable for $X=H$ and $X=V$ and for different $\ell$ using $u_{\text {ref }}^{1}$ an 'eigs'.

The problem is that increasing the number of utilized POD basis functions does not yield better results, it even leads to meaningless results due to the instability of the 'eigs' method. Even if we consider the 'SVD' approach, the error values do not continue to decrease significantly. They somehow stagnate which is of course better than with the 'eigs' method, but still does not yield a satisfying approximation quality. This is due to the fact that the POD basis is chosen poorly. The generated snapshots for basis determination do 
not reflect the dynamics of the optimally controlled trajectory, since the reference control is not chosen well enough. Next, we select a better - somehow

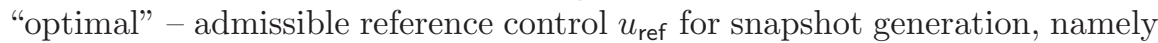
the FE optimal control. The POD basis is now determined with $u_{\text {ref }}^{2}=\bar{u}^{h}$, $X=V$ and snapshots from both the state and the adjoint equation. Using 'SVD' Table 4 presents the deviation of the POD suboptimal controls/states from the FE optimal solutions depending on the number $\ell$ of used POD basis functions. From Table 4 we can conclude that the POD basis should not

\begin{tabular}{r|ccc|cc}
\hline \multicolumn{1}{c|}{$\left\|\zeta^{\ell}\right\| / \gamma$} & $\mathcal{E}_{\mathrm{abs}}^{u}(\ell)$ & $\mathcal{E}_{\mathrm{rel}}^{u}(\ell)$ & $\mathcal{E}_{\mathrm{abs}}^{y}(\ell)$ & $\mathcal{E}_{\mathrm{rel}}^{y}(\ell)$ \\
\hline 1 & $1.1 \cdot 10^{+01}$ & $3.4 \cdot 10^{-00}$ & $6.9 \cdot 10^{-01}$ & $1.7 \cdot 10^{-01}$ & $5.8 \cdot 10^{-02}$ \\
5 & $2.4 \cdot 10^{-00}$ & $1.1 \cdot 10^{-00}$ & $2.2 \cdot 10^{-01}$ & $5.2 \cdot 10^{-02}$ & $1.8 \cdot 10^{-02}$ \\
15 & $2.5 \cdot 10^{-02}$ & $2.5 \cdot 10^{-02}$ & $5.4 \cdot 10^{-03}$ & $3.6 \cdot 10^{-04}$ & $1.2 \cdot 10^{-04}$ \\
20 & $2.8 \cdot 10^{-03}$ & $2.8 \cdot 10^{-03}$ & $6.0 \cdot 10^{-04}$ & $5.1 \cdot 10^{-05}$ & $1.8 \cdot 10^{-05}$ \\
40 & $2.1 \cdot 10^{-06}$ & $2.1 \cdot 10^{-06}$ & $4.5 \cdot 10^{-07}$ & $1.9 \cdot 10^{-08}$ & $6.4 \cdot 10^{-09}$ \\
60 & $8.0 \cdot 10^{-10}$ & $8.0 \cdot 10^{-10}$ & $1.8 \cdot 10^{-10}$ & $5.4 \cdot 10^{-12}$ & $1.9 \cdot 10^{-12}$ \\
70 & $7.0 \cdot 10^{-12}$ & $7.0 \cdot 10^{-12}$ & $1.5 \cdot 10^{-12}$ & $5.0 \cdot 10^{-14}$ & $1.7 \cdot 10^{-14}$ \\
90 & $3.8 \cdot 10^{-13}$ & $1.9 \cdot 10^{-13}$ & $3.7 \cdot 10^{-14}$ & $2.9 \cdot 10^{-14}$ & $9.8 \cdot 10^{-15}$ \\
\hline
\end{tabular}

TABLE 4. Run 4.2: A-posteriori estimator, absolute and relative errors in the control and state variable for $X=V$ and for different $\ell$ using $u_{\text {ref }}^{2}$.

be chosen arbitrarily if we want to obtain a very good approximation quality. The POD based solver with somehow "optimal" reference control $u_{\text {ref }}^{2}$ (Table 4) yields considerably better results than with more or less arbitrary reference control $u_{\text {ref }}^{1}$ (Table 3 ). Table 4 emphasizes the good quality of the POD a-posteriori error estimator. We can also observe that the a posteriori estimator constitutes a reliable upper bound if the 'eigs' approach was taken for basis determination, see Table 5. As long as there is still enough new

\begin{tabular}{c|ccc|ccc}
\hline & & \multicolumn{3}{c}{ 'SVD' } & \multicolumn{3}{c}{ 'eigs' } \\
$\ell$ & $\left\|\zeta^{\ell}\right\| / \gamma$ & $\varepsilon_{\text {abs }}^{u}(\ell)$ & $\frac{\lambda_{\ell}}{\operatorname{tr}\left(\bar{Y} \bar{Y}^{T}\right)}$ & $\left\|\zeta^{\ell}\right\| / \gamma$ & $\varepsilon_{\text {abs }}^{u}(\ell)$ & $\frac{\lambda_{\ell}}{\operatorname{tr}\left(\bar{Y} \bar{Y}^{T}\right)}$ \\
\hline 40 & $2.1 \cdot 10^{-06}$ & $2.1 \cdot 10^{-06}$ & $1.1 \cdot 10^{-14}$ & $2.1 \cdot 10^{-06}$ & $2.1 \cdot 10^{-06}$ & $1.1 \cdot 10^{-14}$ \\
60 & $8.0 \cdot 10^{-10}$ & $8.0 \cdot 10^{-10}$ & $5.1 \cdot 10^{-22}$ & $2.5 \cdot 10^{-08}$ & $2.5 \cdot 10^{-08}$ & $1.7 \cdot 10^{-16}$ \\
70 & $7.0 \cdot 10^{-12}$ & $7.0 \cdot 10^{-12}$ & $8.1 \cdot 10^{-26}$ & $2.0 \cdot 10^{-09}$ & $2.0 \cdot 10^{-09}$ & $8.6 \cdot 10^{-17}$ \\
90 & $3.8 \cdot 10^{-13}$ & $1.9 \cdot 10^{-13}$ & $3.1 \cdot 10^{-30}$ & $2.7 \cdot 10^{-09}$ & $2.5 \cdot 10^{-09}$ & $1.1 \cdot 10^{-16}$ \\
\hline
\end{tabular}

TABLE 5. Run 4.2: A-posteriori estimator, absolute error in the control variable and normalized eigenvalues for $X=V$, for the variants 'SVD' as well as 'eigs' and for different $\ell$ using $u_{\text {ref }}^{2}$.

information content, meaning that the eigenvalues are still "big" enough so that rounding errors do not jeopardize the decreasing order of eigenvalues and the nearly $W$-orthogonality, than the 'eigs' and 'SVD' approaches yield the same eigenvalues and the same suboptimal solutions. This is the case up to $\ell$ scarcely above 40 . From then on, the POD basis determination using 
'eigs' is not stable any more and does not yield approximation errors as good as the 'SVD' approach, see Table 5. Table 6 gives a summary of the CPU times. Notice that $\ell=20$ POD basis functions are sufficient for an approxi-

\begin{tabular}{|c|c|}
\hline & CPU time in $s$ \\
\hline $\begin{array}{l}\text { FE solver } \\
\text { ROM solver }\end{array}$ & 1240.87 \\
\hline $\begin{array}{l}\text { snapshot generation (state \& adjoint) } \\
\text { sna solver }\end{array}$ & 1.62 \\
\hline $\begin{array}{l}\text { POD basis computation with } \ldots \\
\ldots \text { eigs }(\ell=90)\end{array}$ & 1.75 \\
\hline$\ldots \operatorname{SVD}(\ell=90)$ & 6.57 \\
\hline $\begin{array}{l}\text { PDASS incl. assembly of all matrices with } \ldots \\
\ldots \ell=20\end{array}$ & 1.78 \\
\hline$\ldots \ell=90$ & 29.41 \\
\hline a-posteriori error estimation & 2.81 \\
\hline
\end{tabular}

TABlE 6. Run 4.2: CPU times in seconds.

mation of the exact optimal control by the POD suboptimal control, since the POD solutions cannot be significantly better than the FE solution since it is based on FE snapshots and FE matrices. The FE discretization error cannot be overcome by the POD solutions. In case of $\ell=20$ and 'eigs', the overall CPU time needed to compute the POD suboptimal control is 5.15 seconds and thus about 240 times smaller than the CPU time needed to compute the "truth" /FE optimal control.

Finally, we compare the approximation quality of the POD basis if only snapshots from the state (Variant 1) or if snapshots from the state and the adjoint equation (Variant 2) are utilized. Even with "optimal" reference control $u_{\text {ref }}^{2}=\bar{u}^{h}$, snapshots based solely on the state equation are not sufficient for getting good results with the POD ansatz; see Table 7. From Table 7 we

\begin{tabular}{r|c|c|c|c}
\hline & \multicolumn{4}{|c}{$\left\|\bar{u}^{h}-\bar{u}^{\ell}\right\|_{L^{2}(\Sigma)}$} \\
$\ell$ & Variant $2, X=V$ & Variant $1, X=V$ & Variant $1, X=H$ & Variant $2, X=H$ \\
\hline 1 & $3.4 \cdot 10^{-0}$ & $3.4 \cdot 10^{-0}$ & $3.4 \cdot 10^{-0}$ & $3.4 \cdot 10^{-0}$ \\
5 & $1.1 \cdot 10^{-0}$ & $1.1 \cdot 10^{-0}$ & $1.0 \cdot 10^{-0}$ & $9.8 \cdot 10^{-1}$ \\
10 & $1.7 \cdot 10^{-1}$ & $8.8 \cdot 10^{-1}$ & $8.5 \cdot 10^{-1}$ & $5.9 \cdot 10^{-2}$ \\
20 & $2.8 \cdot 10^{-3}$ & $6.5 \cdot 10^{-1}$ & $6.5 \cdot 10^{-1}$ & $1.7 \cdot 10^{-3}$ \\
30 & $1.2 \cdot 10^{-4}$ & $6.0 \cdot 10^{-1}$ & $6.0 \cdot 10^{-1}$ & $9.9 \cdot 10^{-5}$ \\
50 & $1.1 \cdot 10^{-7}$ & $5.1 \cdot 10^{-1}$ & $5.1 \cdot 10^{-1}$ & $2.4 \cdot 10^{-7}$ \\
60 & $2.5 \cdot 10^{-8}$ & $5.0 \cdot 10^{-1}$ & $1.7 \cdot 10^{+2}$ & $1.8 \cdot 10^{-8}$ \\
70 & $2.0 \cdot 10^{-9}$ & $5.0 \cdot 10^{-1}$ & $5.3 \cdot 10^{-1}$ & $2.0 \cdot 10^{-7}$ \\
\hline
\end{tabular}

TABLE 7. Run 4.2: Absolute errors in the control variable for different choices of the snapshot ensemble, for $X$, for 'eigs' and for different $\ell$.

conclude that the inclusion of adjoint information into the snapshot ensemble according to Variant 2 is essential to obtain good approximations for the controls. This is due to the fact that the optimality condition directly relates the control onto the adjoint state variable. Hence, it is important to also capture the dynamics of the adjoint equation in order to have a good snapshot 
ensemble and thus a good POD basis. This coincides with theoretical results, see [10]. The eigenvalues decay slower for $X=V$ than for $X=H$, since there is more information from the snapshot ensemble that gets incorporated into the POD basis. That is why the error values decay slower for small $\ell$. Nevertheless, for higher $\ell$ this higher information content leads to more "stability" and thus monotonously decreasing error values instead of severe oscillations. Summarizing, the choice of $X=V$ and the snapshot ensemble from both the state and the adjoint equation leads to the best performance of the POD-Galerkin ansatz for solving the optimal control problem.

Run 4.3 (Constrained optimal control). We take the same configuration as in Run 4.2 , but now we choose the control constraints $u_{a} \equiv-0.5$ and $u_{b} \equiv 2$. Like in Run 4.2 we make use of the MATLAB PDE toolbox for the spatial discretization with piecewise linear, continuous finite elements with 498 degrees of freedom. For temporal discretization, we use the equidistant time increment $\Delta t=0.004$. The FE solver needs 5 iterations of the primal-dual active set strategy and requires 3435.82 seconds. The optimal control, $\bar{u}^{h}$ is displayed in Figure 5. We now test if the implemented ROM solver works
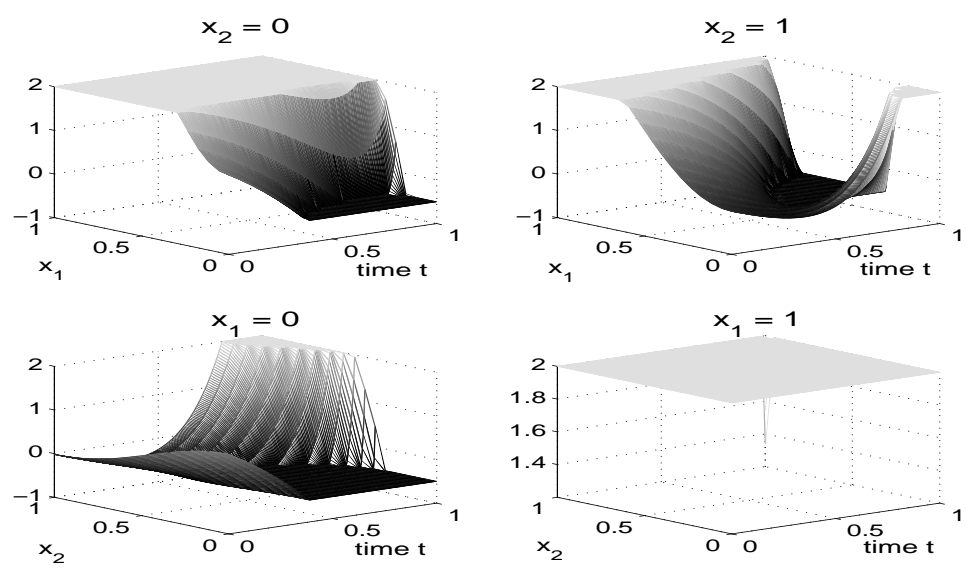

Figure 5. Run 4.3: FE optimal control $\bar{u}^{h}(t, \boldsymbol{x})$ for $\boldsymbol{x}=$ $\left(x_{1}, 0\right) \in \Gamma$ (upper left plot), $\boldsymbol{x}=\left(x_{1}, 1\right) \in \Gamma$ (upper right plot), $\boldsymbol{x}=\left(0, x_{2}\right) \in \Gamma$ (lower left plot), $\boldsymbol{x}=\left(1, x_{2}\right) \in \Gamma$ (lower right plot).

properly when there are active box constraints given for the control $u$. For this, we discuss the results from two different ROM runs. They only vary in the choice of the reference control, first we take the reference control $u_{\text {ref }}^{1}(t, s)=\exp (t)\left(\frac{1}{2}\left|2 x_{1}-x_{2}\right|+\frac{\sin \left(\pi x_{2}\right)}{3}-\frac{1}{3}\right)$ and second we take the FE optimal control $\bar{u}^{h}$ for snapshot generation. In both cases we use $X=V$, the snapshot ensemble from both state and adjoint equation and the 'eigs' method for POD basis computation. 
Note that $u_{\text {ref }}^{1} \notin U_{\text {ad }}$ holds. Nevertheless, we will see that we still get good results, for higher $\ell$ the errors are even smaller than in the unrestricted case with this reference control. In Table 8 we present the POD a-posteriori error estimate, the absolute and the relative error in the control variable for different number $\ell$ of POD basis functions. Taking more POD basis function,

\begin{tabular}{r|ccc|cc}
\hline \multicolumn{1}{c|}{$\ell$} & $\left\|\zeta^{\ell}\right\| / \gamma$ & $\mathcal{E}_{\mathrm{abs}}^{u}(\ell)$ & $\mathcal{E}_{\mathrm{rel}}^{u}(\ell)$ & $\mathcal{E}_{\mathrm{abs}}^{y}(\ell)$ & $\mathcal{E}_{\mathrm{rel}}^{y}(\ell)$ \\
\hline 1 & $1.2 \cdot 10^{+1}$ & $2.2 \cdot 10^{-0}$ & $7.6 \cdot 10^{-1}$ & $1.9 \cdot 10^{-1}$ & $6.7 \cdot 10^{-2}$ \\
5 & $1.3 \cdot 10^{-0}$ & $9.1 \cdot 10^{-1}$ & $3.0 \cdot 10^{-1}$ & $9.6 \cdot 10^{-2}$ & $3.3 \cdot 10^{-2}$ \\
10 & $6.2 \cdot 10^{-1}$ & $3.7 \cdot 10^{-1}$ & $1.3 \cdot 10^{-1}$ & $6.7 \cdot 10^{-2}$ & $2.3 \cdot 10^{-2}$ \\
20 & $5.9 \cdot 10^{-1}$ & $3.2 \cdot 10^{-1}$ & $1.1 \cdot 10^{-1}$ & $3.8 \cdot 10^{-2}$ & $1.3 \cdot 10^{-2}$ \\
30 & $1.2 \cdot 10^{-1}$ & $7.7 \cdot 10^{-2}$ & $2.6 \cdot 10^{-2}$ & $2.1 \cdot 10^{-2}$ & $7.1 \cdot 10^{-3}$ \\
50 & $1.9 \cdot 10^{-2}$ & $1.7 \cdot 10^{-2}$ & $5.5 \cdot 10^{-3}$ & $1.5 \cdot 10^{-2}$ & $5.2 \cdot 10^{-3}$ \\
60 & $1.4 \cdot 10^{-2}$ & $1.2 \cdot 10^{-2}$ & $3.8 \cdot 10^{-3}$ & $1.3 \cdot 10^{-2}$ & $4.3 \cdot 10^{-3}$ \\
70 & $1.2 \cdot 10^{-2}$ & $1.1 \cdot 10^{-2}$ & $3.5 \cdot 10^{-3}$ & $1.2 \cdot 10^{-2}$ & $4.3 \cdot 10^{-3}$ \\
90 & $1.1 \cdot 10^{-2}$ & $9.7 \cdot 10^{-3}$ & $3.2 \cdot 10^{-3}$ & $1.2 \cdot 10^{-2}$ & $4.2 \cdot 10^{-3}$ \\
\hline
\end{tabular}

TABLE 8. Run 4.3: A-posteriori estimator, absolute and relative errors for the control and state variable for $X=V$ and different number $\ell$ of POD basis functions.

into the POD-Galerkin ansatz of the state and adjoint state variable makes the errors for the control variable descend. This behaviour also becomes evident for the absolute deviation of the POD suboptimal state $y^{\ell}$ from the FE optimal state $\bar{y}^{h}$ as well as for the relative error values.

The FE optimal control is restricted by $u_{a}$ at a total amount of 3110 many boundary nodes over all times and prescribed by $u_{b}$ at a total amount of 8410 boundary nodes over all times. In Table 9 we can see how many (diffua) boundary node values are determined by $u_{a}$ in either the POD suboptimal control or in the FE optimal control, but not in both. The differently

\begin{tabular}{c|rrrrrrrrrrrr}
\hline$\ell$ & 5 & 10 & 15 & 20 & 30 & 40 & 50 & 60 & 70 & 80 & 90 & 100 \\
\hline diffua & 998 & 818 & 548 & 596 & 106 & 27 & 24 & 19 & 18 & 18 & 17 & 15 \\
diffub & 700 & 457 & 412 & 415 & 112 & 31 & 33 & 25 & 23 & 23 & 24 & 24 \\
\hline
\end{tabular}

TABLE 9. Run 4.3: Number of differences in restricted node values of the POD suboptimal controls $\bar{u}^{\ell}$ in comparison to the FE optimal control $\bar{u}^{h}$ using $u_{\text {ref }}^{1}$ and $X=V$.

restricted boundary node values by $u_{b}$ are counted and stated in diffub. This table shows that the POD suboptimal control $\bar{u}^{\ell}$ and the FE optimal control $\bar{u}^{h}$ get restricted/fixed equally at an increasing number of boundary nodes with increasing number $\ell$ of POD basis functions used to compute $\bar{u}^{\ell}$. Note that e.g. for $\ell=100$ there are only 15 differences in lower restricted node values meaning that more than $99 \%$ of the $3110 \mathrm{FE}$ restricted values (by $u_{a}$ ) are replicated by the ROM optimal control solver. The same ratio holds true for the values which are fixed by $u_{b}$. 
Now we utilize snapshots generated by the reference control $u_{\text {ref }}=\bar{u}^{h}$. The very good approximation quality can be seen from Table 10. Again, we

\begin{tabular}{r|ccc|cc}
\hline \multicolumn{1}{c|}{$\ell$} & $\left\|\zeta^{\ell}\right\| / \gamma$ & $\mathcal{E}_{\mathrm{abs}}^{u}(\ell)$ & $\mathcal{E}_{\mathrm{rel}}^{u}(\ell)$ & $\mathcal{E}_{\mathrm{abs}}^{y}(\ell)$ & $\mathcal{E}_{\mathrm{rel}}^{y}(\ell)$ \\
\hline 1 & $1.2 \cdot 10^{+1}$ & $2.1 \cdot 10^{+0}$ & $7.20 \cdot 10^{-1}$ & $1.4 \cdot 10^{-1}$ & $4.9 \cdot 10^{-2}$ \\
5 & $6.5 \cdot 10^{-1}$ & $5.6 \cdot 10^{-1}$ & $1.88 \cdot 10^{-1}$ & $2.3 \cdot 10^{-2}$ & $7.9 \cdot 10^{-3}$ \\
15 & $2.7 \cdot 10^{-2}$ & $2.7 \cdot 10^{-2}$ & $9.0 \cdot 10^{-3}$ & $7.12 \cdot 10^{-4}$ & $2.5 \cdot 10^{-4}$ \\
20 & $7.5 \cdot 10^{-3}$ & $7.3 \cdot 10^{-3}$ & $2.4 \cdot 10^{-3}$ & $1.97 \cdot 10^{-4}$ & $6.9 \cdot 10^{-5}$ \\
40 & $3.9 \cdot 10^{-4}$ & $3.9 \cdot 10^{-4}$ & $1.3 \cdot 10^{-4}$ & $8.09 \cdot 10^{-6}$ & $2.8 \cdot 10^{-6}$ \\
60 & $8.3 \cdot 10^{-5}$ & $8.3 \cdot 10^{-5}$ & $2.8 \cdot 10^{-5}$ & $1.61 \cdot 10^{-6}$ & $5.6 \cdot 10^{-7}$ \\
70 & $3.0 \cdot 10^{-5}$ & $3.0 \cdot 10^{-5}$ & $1.0 \cdot 10^{-5}$ & $6.02 \cdot 10^{-7}$ & $2.1 \cdot 10^{-7}$ \\
90 & $3.7 \cdot 10^{-6}$ & $3.7 \cdot 10^{-6}$ & $1.3 \cdot 10^{-6}$ & $1.21 \cdot 10^{-7}$ & $4.2 \cdot 10^{-8}$ \\
130 & $3.9 \cdot 10^{-8}$ & $3.9 \cdot 10^{-8}$ & $1.3 \cdot 10^{-8}$ & $4.49 \cdot 10^{-9}$ & $1.6 \cdot 10^{-9}$ \\
\hline
\end{tabular}

TABLE 10. Run 4.3: A-posteriori estimator, absolute and relative errors for the control and state variable for $X=V$ and different number $\ell$ of POD basis functions.

check if the values of the POD suboptimal control are restricted at the same boundary nodes as the FE optimal control and how this changes depending on the number $\ell$ of used POD basis functions, see Table 11. The active sets

\begin{tabular}{c|rrrrrrrrrrrrrrr}
\hline$l$ & 5 & 10 & 15 & 20 & 30 & 40 & 50 & 60 & 70 & 80 & 90 & 100 & 110 & 120 & 130 \\
\hline diffua & 1021 & 311 & 74 & 28 & 5 & 2 & 0 & 0 & 0 & 0 & 0 & 0 & 0 & 0 & 0 \\
diffub & 324 & 147 & 37 & 8 & 0 & 0 & 0 & 0 & 0 & 0 & 0 & 0 & 0 & 0 & 0 \\
\hline
\end{tabular}

TABLE 11. Run 4.3: Number of differences in restricted node values of the POD suboptimal controls $\bar{u}^{\ell}$ in comparison to the FE optimal control $\bar{u}^{h}$ using $u_{\text {ref }}^{2}$ and $X=V$.

within the reduced order method coincide with those within the FE approach for $\ell \geq 50$. Compared to the results associated with $u_{\text {ref }}^{1}$ in Table 9 , the more prudent choice of the reference control and the POD basis leads to more/earlier harmony of restricted values.

\section{References}

[1] K. Afanasiev and M. Hinze, Adaptive control of a wake flow using proper orthogonal decomposition. Lecture Notes in Pure and Applied Mathematics, 216 (2001), 317-332.

[2] E. Arian, M. Fahl, and E.W. Sachs, Trust-region proper orthogonal decomposition for flow control. Technical Report 2000-25, ICASE, 2000.

[3] R. Dautray and J.-L. Lions, Mathematical Analysis and Numerical Methods for Science and Technology. Volume 5: Evolution Problems I. Springer, Berlin, 1992.

[4] P. Benner and E.S. Quintana-Ortí, Model reduction based on spectral projection methods. In Reduction of Large-Scale Systems, P. Benner, V. Mehrmann, D.C. Sorensen (eds.), Lecture Notes in Computational Science and Engineering, 45 (2005), 5-48. 
[5] L. Dede, Reduced basis method and a posteriori error estimation for parameterized optimal control problems. SIAM J. Sci., 32 (2010), 997-1019.

[6] M.A. Grepl and M. Kärcher. Reduced basis a posteriori error bounds for parametrized linear-quadratic elliptic optimal control problems. C. R. Acad. Sci. Paris, Ser. I, 349 (2011) 873-877.

[7] M. Hintermüller, K. Ito, and K. Kunisch, The primal-dual active set strategy as a semi-smooth Newton method. SIAM J. Opt., 13 (2003), 865-888.

[8] M. Hintermüller, I. Kopacka, and S. Volkwein, Mesh-independence and preconditioning for solving parabolic control problems with mixed control-state constraints. ESAIM: COCV, 15 (2008), 626-652.

[9] M. Hintermüller and M. Ulbrich, A mesh-independence result for semismooth Newton methods. Math. Program. Ser. B, 101 (2004), 151-184.

[10] M. Hinze and S. Volkwein, Error estimates for abstract linear-quadratic optimal control problems using proper orthogonal decomposition. Computat. Optim. and Appl., 39 (2008), 319-345.

[11] P. Holmes, J.L. Lumley, and G. Berkooz, Turbulence, Coherent Structures, Dynamical Systems and Symmetry. Cambridge Monographs on Mechanics, Cambridge University Press, 1996.

[12] M. Kahlbacher and S. Volkwein, POD a-posteriori error based inexact SQP method for bilinear elliptic optimal control problems. ESAIM: M2AN, to appear.

[13] E. Kammann, F. Tröltzsch and S. Volkwein, A method of a-posteriori error estimation with application to proper orthogonal decomposition. (2011), submitted.

[14] K. Kunisch and S. Volkwein, Galerkin proper orthogonal decomposition methods for a general equation in fluid dynamics. SIAM J. Numer. Anal., 40 (2002), 492515 .

[15] K. Kunisch and S. Volkwein, Proper orthogonal decomposition for optimality systems. ESAIM: M2AN, 42 (2008), 1-23.

[16] J.L. Lions, Optimal Control of Systems Governed by Partial Differential Equations. Springer, Berlin, 1971.

[17] A. Manzoni, A. Quarteroni, and G. Rozza, Shape optimization for viscous flows by reduced basis methods and free-form deformation. Int. J. Numer. Meth. Fluids (2011), to appear.

[18] B. Noble, Applied Linear Algebra. Englewood Cliffs, Prentice-Hall, 1969.

[19] J. Nocedal and S.J. Wright. Numerical Optimization. Springer Series in Operation Research, Second Edition (2006).

[20] E. Sachs and S. Volkwein, POD-Galerkin approximations in PDE constrained optimization. GAMM-Mitt., 33 (2010), 194-208.

[21] T. Tonn, K. Urban, and S. Volkwein, Comparison of the reduced-basis and POD a-posteriori error estimators for an elliptic linear quadratic optimal control problem. Math. and Comp. Modelling of Dyn. Systems, 17 (2011), 355-369.

[22] F. Tröltzsch and S. Volkwein, POD a-posteriori error estimates for linearquadratic optimal control problms. Comput. Optim. Appl. 44 (2009), 83-115.

[23] S. Volkwein, Model Reduction using Proper Orthogonal Decomposition. Lecture Notes, University of Constance (2010). 
[24] S. Volkwein, Optimality system POD and a-posteriori error analysis for linearquadratic problems. Submitted (2010).

[25] G. Vossen and S. Volkwein, Model reduction techniques with a-posteriori error analysis for linear-quadratic optimal control problems. Submitted (2011).

\author{
Alina Studinger \\ University of Constance \\ Department of Mathematics and Statistics \\ Universitätsstraße 10 \\ D-78457 Konstanz \\ Germany \\ e-mail: astudinger@web.de \\ Stefan Volkwein \\ University of Constance \\ Department of Mathematics and Statistics \\ Universitätsstraße 10 \\ D-78457 Konstanz \\ Germany \\ e-mail: Stefan.Volkwein@uni-konstanz.de
}

